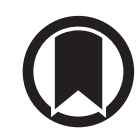

CrossMark

\title{
Mitochondrial dysfunction in macrophages: a key to defective bacterial phagocytosis in COPD
}

\author{
Mathew Suji Eapen (10), Pawan Sharma $\mathbb{1}^{1,2}$ and Sukhwinder Singh Sohal ${ }^{1}$ \\ Affiliations: ${ }^{1}$ Respiratory Translational Research Group, Dept of Laboratory Medicine, School of Health \\ Sciences, College of Health and Medicine, University of Tasmania, Launceston, Australia. ${ }^{2}$ Center for \\ Translational Medicine, Thomas Jefferson University, Philadelphia, PA, USA.
}

Correspondence: Sukhwinder Singh Sohal, Respiratory Translational Research Group, Dept of Laboratory Medicine, School of Health Sciences, College of Health and Medicine, University of Tasmania, Locked Bag 1322, Newnham Drive, Launceston, Tasmania 7248, Australia. E-mail: sssohaldautas.edu.au

@ERSpublications

Pulmonary macrophages are key to elimination of respiratory pathogens. Oxidative stress and mitochondrial dysfunction are associated with defective macrophage phagocytosis in COPD, with implications for aberrant cellular metabolism and early cell death. http://bit.ly/2m2uA0z

Cite this article as: Eapen MS, Sharma P, Sohal SS. Mitochondrial dysfunction in macrophages: a key to defective bacterial phagocytosis in COPD. Eur Respir J 2019; 54: 1901641 [https://doi.org/10.1183/ 13993003.01641-2019].

COPD is now well recognised as a complex, devastating disease which affects the lungs of tobacco smokers in developed nations, though the contribution to the disease by biomass and fossil fuel burning cannot be ruled out, especially in less advanced or third world nations [1,2]. An estimated 250 million people are affected worldwide as per 2016 estimates, with 3.17 million deaths annually, which is $5 \%$ of total worldwide mortality [3]. COPD stands to be the third-largest cause of death worldwide, and is often associated with respiratory infections, comorbidities and lung cancer, which further increases the risk of mortality [4]. The disease is characterised as slowly progressive with partial reversibility at most, with some pathological changes evident even after smoking cessation [5]. COPD patients develop structural changes, which leads to small airway wall thickening, narrowing and, ultimately, obliteration [6,7]. The large airway pathology includes squamous cell metaplasia, mucus hypersecretion, and smooth muscle hyperplasia accompanied by chronic bronchitis $[8,9]$.

Further, close to $50 \%$ of patients suffer from a varying degree of emphysematous lung destruction, which also adds to airflow limitation and disease symptoms [10]. In more severe COPD, patients experience acute exacerbations, which are precipitated by a significant increase in aberrant inflammation and respiratory tract infections. Acute exacerbations in COPD patients are associated with accelerated loss of lung function, increased mortality, decreased health-related quality of life, and cause substantial economic burden to society [8]. Bacterial pathogens constitute a significant proportion of acute exacerbations of COPD, with nontypeable Haemophilus influenzae (NTHi) being the most common bacterium, followed by Streptococcus pneumoniae and Moraxella catarrhalis.

Microbial infections frequently cause severe pneumonia in susceptible COPD patients. These infectious pathogenicities are often observed to be further aggravated by increase in viral co-infections. Although a robust pulmonary immune defence system exists, involving a highly evolved mechanism of both innate and adaptive immunity, under pathological conditions, microbes often manage to defend or evade host 
immune response, increasing the bacterial load and colonisation in the tissue [8]. One possible evasive mechanism employed by microbes is increased adherence to airway epithelial cells through receptors such as platelet-activating factor receptor and intracellular adhesion molecule-1 $[8,11-13]$.

Inflammation forms a crucial factor in COPD and is known to variably affect patients based on their disease stage and age. However, more recently, the effectiveness of immune cell function has been called into question, with several studies indicating both dysfunctionality and dysregulation in immune cells [14-17]. Further, the cellular profile in airway tissue has been somewhat paradoxical; although literature evidence demonstrates a consistent increase in airway inflammatory cells in the lumen, the overall picture in the airway wall has been variable across laboratories for several years [18]. Indeed, our recent observation suggested a decrease in both total cellularity and key inflammatory cells in the airway wall of mildmoderate COPD patients compared to smokers and healthy controls, which indicate possible immune suppression and thus increased susceptibility to infections, at least early in the disease [19].

Pulmonary macrophages are important players in lung immunity, constituting about 80 to 90 percent of the total immune cell population. Two distinct phenotypes of macrophages have been identified in the lung; one is smaller and resides in the airway wall or is associated with the interstitium, while the other is the larger alveolar macrophage, which lies within the alveolar spaces close to the lung parenchyma. Based on their localisation, macrophages play a crucial role in maintaining lung homeostasis, immune surveillance, and microbial and cellular clearance, among others [20]. Several studies in the past have shown specific impairment of macrophages in COPD patients leading to reduced phagocytosis or efferocytosis [14]; however, the inherent mechanisms by which this could occur is not established.

The study by BelChamber et al. [21] published in the current issue of the European Respiratory Journal tries to systematically elucidate the mechanism that causes macrophage functional impairment and identifies the contribution of mitochondrial dysfunction in augmenting such changes. The authors performed several in vitro phagocytic assays on respiratory bacterium Streptococcus pneumoniae and NTHi, using isolated alveolar macrophages and monocyte-derived macrophages (MDMs) that were differentiated either by the granulocyte-macrophage colony-stimulating factor (GM-CSF) or macrophage colony-stimulating factor (M-CSF).

Interestingly, the authors found that stimulation with either GM-CSF or M-CSF did not alter phagocyting macrophage numbers in smokers or COPD compared to healthy controls. However, phagocytic efficiency was severely attenuated in COPD patients. This decrease in macrophage efficiency was observed in smokers, albeit to a lesser extent than in COPD, suggesting a smoking effect driving such dysfunctionality and further exaggerated in COPD. Both GM-CSF and M-CSF are members of colony-stimulating factor superfamily and are primarily involved in the generation of mammalian myelopoiesis namely associated with the production of macrophages, dendritic cells and polymorphonuclear phagocytes [22]. While $\mathrm{M}$-CSF is implicated for steady-state control of tissue macrophage development and differentiation into its phagocytic forms, GM-CSF is often known to produce and act locally at sites of tissue inflammation [22]. Such differential changes are evident from the authors' data (in their figure 2), where a two-fold increase is observed in bacterial phagocytosis among non-smoker M-CSF derived MDMs over GM-CSF. Both GM-CSF and M-CSF are also known to induce opposite responses in macrophages, with GM-CSF stimulating a more inflammatory M1 while M-CSF pushing them towards a more non-inflammatory, pro-proliferative M2 phenotype. Previous reports confirm that human alveolar macrophages are indeed more M2 in nature with upregulation of genes associated with scavenger receptor such as CD36 and CD163, both upregulated by M-CSF induction [23]. A recent study from our laboratory also demonstrated M1 to M2 macrophage switch in bronchioalveolar lavage from smokers and mild-moderate COPD patients with a concomitant M2 cytokine/chemokine profile. We further observed a substantial decrease in inducible nitric oxide synthase expression in these patients compared to healthy controls, which again points towards macrophage dysfunctionality [24].

Inefficient phagocytosis by macrophages poses an important question about whether macrophages from COPD suffer from energy deficit required to perform their regular task of bacterial killing. The authors thus investigated the role of mitochondria in COPD macrophages by measuring changes in mitochondria reactive oxygen species (mROS) and mitochondrial membrane potential. Recent evidence has linked increased oxidative stress in COPD patients to mROS production and mitochondrial DNA damage [25, 26]. The authors observed that COPD macrophages that engulfed respiratory bacteria showed both an increase in mROS species and a decrease in mitochondrial membrane potential. Such changes, however, differed based on the type of macrophage induction and microbial infection used in the study. Interestingly, NTHi infections and phagocytosis specifically affected mitochondrial membrane potential in COPD patients compared to Streptococcus pneumoniae in both M-CSF and GM-CSF MDMs. Loss of the mitochondrial membrane potential is known to cause energy failure in the cells, proton leakages, increase 
in mROS, and eventual cell death [27]. Such loss in membrane potential have previously been attributed to increase in release of cytochrome $\mathrm{c}$ from mitochondria into cytoplasm, which activates caspases cascade pathways causing cellular apoptosis [28]. This study thus, possibly for the first time in respiratory bacteria, provides evidence on the differential mechanism used by microbes in achieving their desired infectious objectives. Besides, more importantly, these infections are now known to affect the energy balance in macrophages, causing their observed dysfunctionality.

Interestingly, but not entirely surprisingly, external addition of stress inducers, such as $\mathrm{H}_{2} \mathrm{O}_{2}$, inhibited the capacity of alveolar macrophages and MDMs to phagocytose microbes, and thus further characterises dysfunctionality. Reactive oxygen species generated via cigarette smoke extract exposure have previously been demonstrated in epithelial cells to produce mitochondrial dysfunction displayed by loss of adequate energy generation via ATP, reduced mitochondrial membrane potential, and an overall decrease in mitochondrial oxygenation [29]. Further, smokers are potentially vulnerable to an increase in mitochondrial DNA damage, with several toxins associated with tobacco smoke involved in tissue damage. Indeed, previous studies on alveolar macrophages isolated from COPD patients indicate that mitochondrial DNA is more vulnerable to tobacco smoke insults than nuclear DNA, which could lead to decrease in oxidative phosphorylation, an increase in free radical generation and decrease in ATP (energy production) [25]. The overall decline in energy could be spontaneously attributed to early ageing of the cells and push towards cellular senescence [30].

Currently, very few treatments exist that could affect the overall course of COPD; existing therapies at most provide symptomatic relief and help reduce exacerbation rates. The current use of immune-suppressive drugs, such as corticosteroids, in the treatment regimen could increase the frequency of microbial infections in COPD patients, which are potentially harmful in the long run [11]. Understanding fundamental mechanisms associated with respiratory infections is vital for underpinning the pathogenicity and to provide opportunities for exploring new target-specific therapeutic agents. Findings in this study provide several unique insights into the fundamental dysfunctionality of airway macrophages in phagocytosing airway pathogens and the vital role played by differentiating factors in influencing such outcomes. Also, of great interest is the implication associated with alteration in mitochondrial dynamics in response to bacterial infections. Changes in mitochondrial energetics, mitochondrial DNA damage, and an increase in mROS production, will have several consequential cellular effects, including structural changes, metabolism and cell death. Restoring mitochondrial dysfunction could be a potential therapeutic target for combating microbial pathogenesis in COPD.

Acknowledgements: S.S. Sohal is supported by Clifford Craig Foundation Launceston General Hospital, Rebecca L. Cooper Medical Research Foundation, Lung Foundation Australia, Cancer Council Tasmania, Thoracic Society of Australia and New Zealand (TSANZ) and Boehringer Ingelheim. P. Sharma is supported by Rebecca L. Cooper Medical Research Foundation, Australia.

Conflict of interest: None declared.

\section{References}

1 Rhee CK, Chau NQ, Yunus F, et al. Management of COPD in Asia: a position statement of the Asian Pacific Society of Respirology. Respirology 2019; 24: 1018-1025.

2 Salvi S KG, Dhaliwal RS, Paulson K, et al. The burden of chronic respiratory diseases and their heterogeneity across the states of India: the Global Burden of Disease Study 1990-2016. Lancet Glob Health 2018; 6: e1363-e1374.

3 World Health Organization. Chronic Obstructive Pulmonary Disease (COPD). Date last updated: 1 December 2017. www.who.int/news-room/fact-sheets/detail/chronic-obstructive-pulmonary-disease-(copd)

4 World Health Organization. The Top 10 Causes of Death. Date last updated: 24 May 2018. www.who.int/ news-room/fact-sheets/detail/the-top-10-causes-of-death

5 Willemse BWM, Postma DS, Timens W, et al. The impact of smoking cessation on respiratory symptoms, lung function, airway hyperresponsiveness and inflammation. Eur Respir J 2004; 23: 464-476.

6 Hogg JC, Paré PD, Hackett T-L. The contribution of small airway obstruction to the pathogenesis of chronic obstructive pulmonary disease. Physiol Rev 2017; 97: 529-552.

7 Koo HK, Vasilescu DM, Booth S, et al. Small airways disease in mild and moderate chronic obstructive pulmonary disease: a cross-sectional study. Lancet Respir Med 2018; 6: 591-602.

8 Atto B, Eapen MS, Sharma P, et al. New therapeutic targets for the prevention of infectious acute exacerbations of COPD: role of epithelial adhesion molecules and inflammatory pathways. Clin Sci 2019; 133: 1663-1703.

9 Peters EJ, Morice R, Benner SE, et al. Squamous metaplasia of the bronchial mucosa and its relationship to smoking. Chest 1993; 103: 1429-1432.

10 McDonough JE, Yuan R, Suzuki M, et al. Small-airway obstruction and emphysema in chronic obstructive pulmonary disease. N Engl J Med 2011; 365: 1567-1575.

11 Sohal SS. Inhaled corticosteroids and increased microbial load in COPD: potential role of epithelial adhesion molecules. Eur Respir J 2018; 51: 1702257.

12 Grigg J. The platelet activating factor receptor: a new anti-infective target in respiratory disease? Thorax 2012; 67: $840-841$. 
13 Grigg J, Walters H, Sohal SS, et al. Cigarette smoke and platelet-activating factor receptor dependent adhesion of Streptococcus pneumoniae to lower airway cells. Thorax 2012; 67: 908-913.

14 Hiemstra PS. Altered macrophage function in chronic obstructive pulmonary disease. Ann Am Thorac Soc 2013; 10: S180-S185.

15 Ghosh B, Gaike AH, Pyasi K, et al. Bacterial load and defective monocyte-derived macrophage bacterial phagocytosis in biomass smoke-related COPD. Eur Respir J 2019; 53: 1702273.

16 McKendry RT, Spalluto CM, Burke H, et al. Dysregulation of antiviral function of CD8(+) T cells in the chronic obstructive pulmonary disease lung. Role of the PD-1-PD-L1 axis. Am J Respir Crit Care Med 2016; 193: 642-651.

17 Singh D. Reduced antibacterial immunity in chronic obstructive pulmonary disease: a key role for lymphocytes? Am J Respir Crit Care Med 2014; 190: 3-4.

18 O'Donnell R, Breen D, Wilson S, et al. Inflammatory cells in the airways in COPD. Thorax 2006; 61: 448-454.

19 Eapen MS, McAlinden K, Tan D, et al. Profiling cellular and inflammatory changes in the airway wall of mild to moderate COPD. Respirology 2017; 22: 1125-1132.

20 Byrne AJ, Mathie SA, Gregory LG, et al. Pulmonary macrophages: key players in the innate defence of the airways. Thorax 2015; 70: 1189-1196.

21 Belchamber KBR, Singh R, Batista CM, et al. Defective bacterial phagocytosis is associated with dysfunctional mitochondria in COPD macrophages. Eur Respir J 2019; 54: 1802244.

22 Becher B, Tugues S, Greter M. GM-CSF: from growth factor to central mediator of tissue inflammation. Immunity 2016; 45: 963-973.

23 Shaykhiev R, Krause A, Salit J, et al. Smoking-dependent reprogramming of alveolar macrophage polarization: implication for pathogenesis of chronic obstructive pulmonary disease. J Immunol 2009; 183: 2867-2883.

24 Eapen MS, Hansbro PM, McAlinden K, et al. Abnormal M1/M2 macrophage phenotype profiles in the small airway wall and lumen in smokers and chronic obstructive pulmonary disease (COPD). Sci Rep 2017; 7: 13392.

25 Ballinger SW, Bouder TG, Davis GS, et al. Mitochondrial genome damage associated with cigarette smoking. Cancer Res 1996; 56: 5692-5697.

26 Ryter SW, Rosas IO, Owen CA, et al. Mitochondrial dysfunction as a pathogenic mediator of chronic obstructive pulmonary disease and idiopathic pulmonary fibrosis. Ann Am Thorac Soc 2018; 15: S266-S272.

27 Zamzami N, Marchetti P, Castedo M, et al. Sequential reduction of mitochondrial transmembrane potential and generation of reactive oxygen species in early programmed cell death. J Exp Med 1995; 182: 367-377.

28 Liu X, Kim CN, Yang J, et al. Induction of apoptotic program in cell-free extracts: requirement for dATP and cytochrome c. Cell 1996; 86: 147-157.

29 Ballweg K, Mutze K, Königshoff M, et al. Cigarette smoke extract affects mitochondrial function in alveolar epithelial cells. Am J Physiol Lung Cell Mol Physiol 2014; 307: L895-L907.

30 Birch J, Passos JF. Targeting the SASP to combat ageing: mitochondria as possible intracellular allies? Bioessays 2017; 39: 10.1002/bies.201600235. 